\title{
The Use of “Scalp Block” in Pediatric Patients
}

\author{
Joseph Sebeo, Irene P. Osborn
}

Department of Anesthesiology, Mount Sinai School of Medicine, New York, USA.

Email: joseph.sebeo@mssm.edu

Received March $8^{\text {th }}$, 2012; revised April 15 ${ }^{\text {th }}$, 2012; accepted May $12^{\text {th }}, 2012$

\begin{abstract}
Infiltration of the nerves of the scalp with local anesthetics is used in adults for a variety of head and neck procedures and craniotomies with many benefits, from hemodynamic stability to reduced postoperative pain. We here succinctly review the current evidence for "scalp block" in pediatric patients.
\end{abstract}

Keywords: Local Anesthetics; Scalp Block; Pediatric; Anesthesia; Analgesia

\section{Introduction}

Regional anesthesia is becoming increasingly popular in children from neuraxial blocks to peripheral nerve blockade $[1,2]$. Regional anesthesia for pediatric procedures may be performed in the sedated patient [3], although expert panels including the UK Epidural Audit, the French Language Study of Regional anesthetics, and the Pediatric Regional Anesthetic Network (PRAN) all support regional anesthesia in asleep children as a safer approach [4,5]. The "scalp block" technique is an underutilized regional anesthetic technique, which is relatively easy to perform with great benefits to patients [6]. It is a natural evolution that the "scalp block", well-described for adult craniotomies [6,7], be extended to children. We here provide a brief review of the use of this technique in the pediatric population.

\section{Anatomy of "Scalp Block" in Children}

The modern "scalp block" in adults is classically described with potential blockade of six nerves including the supraorbital, supratrochlear, auriculotemporal, zygomaticotemporal, greater occipital and lesser occipital nerves [6,8-12]. This technique has been adapted to children with blocking of both the anterior (supraorbital and supratrocheal nerves) and posterior scalp (greater occipital nerve) with bupivicaine. Figure 1 displays a schematic of scalp nerves in a pediatric patient. Of note, ropivacaine or levobupivacaine have also been utilized for these blocks, but to date no data support the use of one anesthetic over the other.

\subsection{Anterior Scalp}

1) The supraorbital nerve (see Figure 2): the supraorbital notch is palpated by the finger along the supe- rior orbital rim, and the needle is inserted along the upper orbital margin, perpendicular to the skin, approximately $1 \mathrm{~cm}$ medial to the supraorbital foramen. The current literature supports using $1-1.5 \mathrm{ml}$ of $0.25 \%$ bupivicaine with 1:200,000 epinephrine in children [8,9].

2) The supratrochlear nerve (see Figure 2): the supratrochlear nerve can be blocked by medial extension of the supraorbital block as it emerges above the eyebrow. Recommendation for children is $1 \mathrm{ml}$ of $0.25 \%$ bupivacaine with frequent aspirations to prevent intravascular injection $[8,9]$.

\subsection{Posterior Scalp}

The greater occipital nerve (see Figure 3): this nerve can be blocked by infiltration approximately halfway between the occipital protuberance and the mastoid process, with gradual injection medially to the occipital artery after careful aspiration. The reported dosage for pediatric patients is 2 - $3 \mathrm{ml}$ of $0.25 \%$ bupivacaine with $1: 200,000$ epinephrine [8-10].

Of note, there have been rare reports of ultrasound use to perform isolated nerve block of the scalp, but no advantage has been established to date over the described classic landmark technique for "scalp block” [11].

\section{Pediatric “Scalp Block" Today and Tomorrow}

"Scalp blocks" have been performed in children for several years. As with adults, pain increases morbidity in the postoperative period in both neonates and children [12, 13], and adequate pain management in children is an ongoing effort [14]. The use of scalp block in children may include several benefits including decreased adverse side effects of high dose-opioids (nausea, itching, respi- 


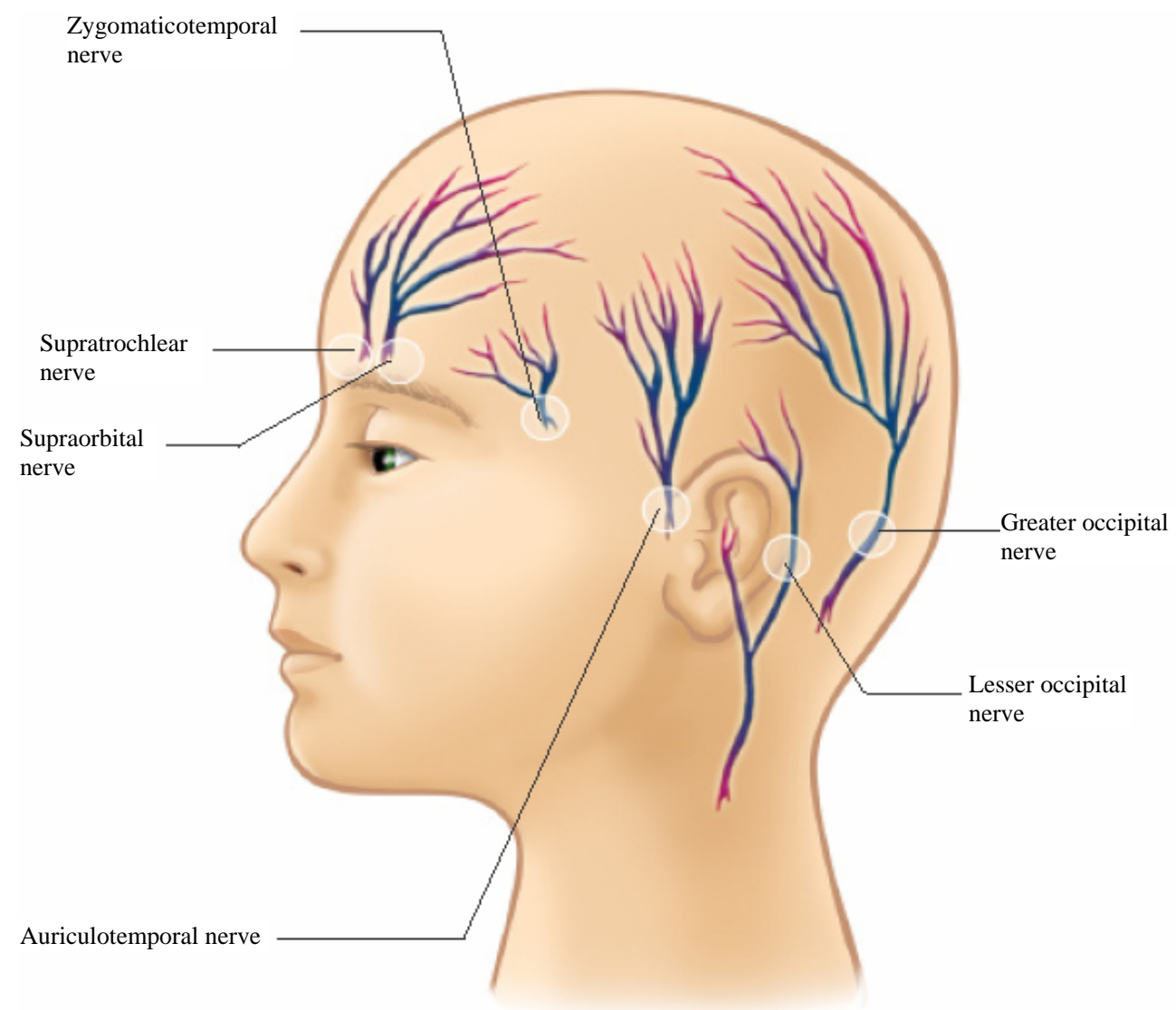

Figure 1. Schematic of scalp block nerves in a pediatric patient.

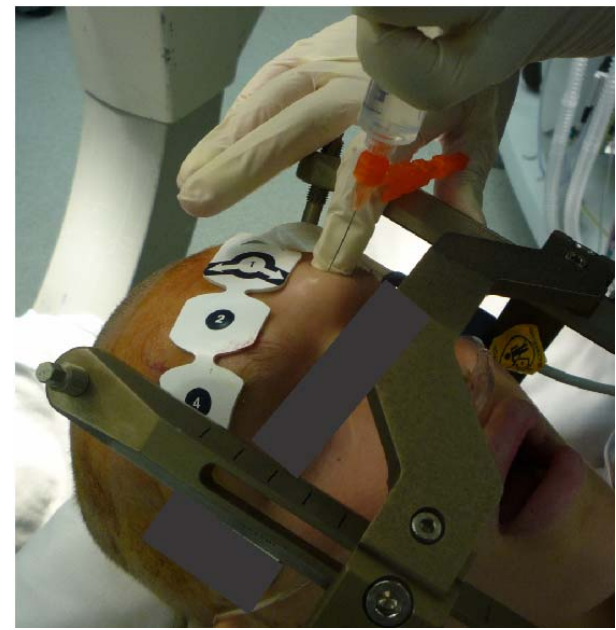

Figure 2. Supraorbital nerve block in a pediatric patients.

ratory depression) [10], and postoperative pain relief with the use of long-acting local anesthetics. Use of scalp block also results in hemodynamic stability: mean arterial pressure (MAP) and heart rate (HR) responses to scalp incision and reflection are attenuated by scalp infiltration in pediatric patients undergoing craniotomy [15].

"Scalp block" with $0.25 \%$ bupivacaine with epineph-

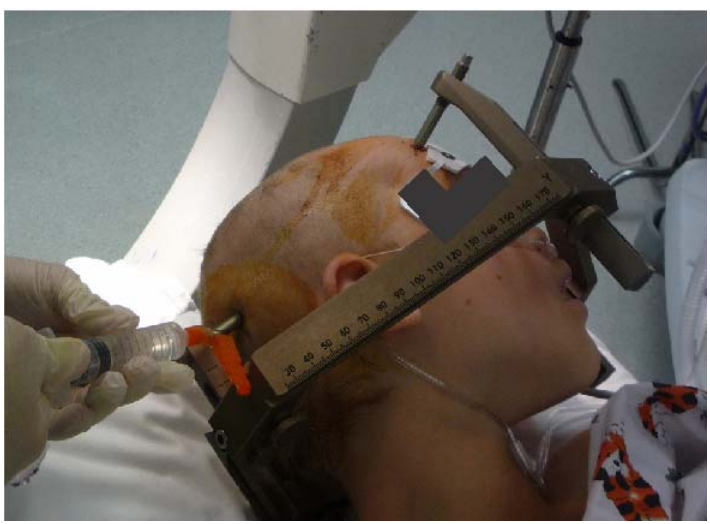

Figure 3. Greater occipital nerve block in a pediatric patients.

rine (greater auricular nerve) has successfully been used in children undergoing tympanomastoid surgery with advantages including avoidance of the adverse side effects of opioids, and targeting the pain relief to the area of the lesion $[10,16]$. Greater auricular nerve block was thus shown to greatly reduce the overall opioid requirements in children undergoing tympanomastoid surgery [10].

The supraorbital and supratrochlear nerve have successfully been blocked in children undergoing frontal 
craniotomies, nevus scalp removal, and midline dermoid excision [8,9]. The "scalp block" technique in children has recently been extended with block of the supraorbital, supratrochlear, auriculotemporal, lesser occipital and greater occipital nerves in a pediatric patient undergoing craniotomy [17]. This block was used as an adjunct to propofol/remifentanil based anesthetic during awake craniotomy and tumor resection in an adolescent patient [17]. Infiltration of the supraorbital, supratrochlear, auriculotemporal, greater auricular, greater occipital, and lesser occipital in an 8-year-old girl undergoing a thalamic tumor resection with levobupivicaine $0.25 \%$, was also recently shown to prevent the hemodynamic response to skull-pin placement and decrease the need for opioids [18]. This "extended scalp block" (infiltration of the supraorbital, supratrochlear, auriculotemporal, and posterior auricular nerves) in patients undergoing neurovascular reconstruction surgery resulted in more stable hemodynamic parameters, with lower concentrations of inhaled anesthetics (sevoflurane) needed, and decreased morbidity compared to the control group [19].

Further expansions of this "scalp block" technique in children have started to include pediatric patients undergoing craniosynostosis repair and awake procedures for deep brain stimulation for dystonia [20,21].

\section{Conclusion}

Regional anesthesia has often combined with general anesthesia to provide patients with a synergistic multimodal approach to analgesia and hemodynamic stability [22]. Similarly to its use in adults, the "scalp block" may be used in children undergoing a variety of procedures, from neurosurgical to otorhinolaryngological procedures. The utilization of local nerve blocks of the scalp in children may thus provides analgesia with stable hemodynamics while reducing the need for other anesthetics such as inhaled anesthetics and opioids: this could in turn result in less side effects, higher patient and family satisfaction, and better outcomes.

\section{REFERENCES}

[1] B. C. Tsui and S. Suresh, "Ultrasound Imaging for Regional Anesthesia in Infants, Children, and Adolescents: A Review of Current Literature and Its Application in the Practice of Neuraxial Blocks,” Anesthesiology, Vol. 112, No. 3, 2010, pp. 719-728. doi:10.1097/ALN.0b013e3181c5e03a

[2] V. C. Ponde, "Continuous Infraclavicular Brachial Plexus Block: A Modified Technique to Better Secure Catheter Position in Infants and Children,” Anesthesia \& Analgesia, Vol. 106, No. 1, 2008, pp. 94-96. doi:10.1213/01.ane.0000289633.81407.65

[3] P. Marhofer, et al., "Ultrasound Guidance for Infraclavicular Brachial Plexus Anaesthesia in Children,” Anaes- thesia, Vol. 59, No. 7, 2004, pp. 642-646. doi:10.1111/j.1365-2044.2004.03669.x

[4] N. Llewellyn and A. Moriarty, "The National Pediatric Epidural Audit,” Pediatric Anesthesia, Vol. 17, No. 6, 2007, pp. 520-533. doi:10.1111/j.1460-9592.2007.02230.x

[5] E. Giaufre, B. Dalens and A. Gombert, "Epidemiology and Morbidity of Regional Anesthesia in Children: A OneYear Prospective Survey of the French-Language Society of Pediatric Anesthesiologists,” Anesthesia \& Analgesia, Vol. 83, No. 5, 1996, pp. 904-912. doi:10.1213/00000539-199611000-00003

[6] I. Osborn and J. Sebeo, “'Scalp Block’ during Craniotomy: A Classic Technique Revisited,” Journal of Neurosurgical Anesthesiology, Vol. 22, No. 3, 2010, pp. 187194. doi:10.1097/ANA.0b013e3181d48846

[7] T. G. Costello and J. R. Cormack, "Anaesthesia for Awake Craniotomy: A Modern Approach,” Journal of Clinical Neuroscience, Vol. 11, No. 1, 2004, pp. 16-19. doi:10.1016/j.jocn.2003.09.003

[8] S. Suresh and A. M. Wagner, "Scalp Excisions: Getting 'Ahead' of Pain,” Pediatric Dermatology, Vol. 18, No. 1, 2001, pp. 74-76. doi:10.1046/j.1525-1470.2001.018001074.x

[9] S. Suresh and P. Voronov, "Head and Neck Blocks in Children: An Anatomical and Procedural Review," Pediatric Anesthesia, Vol. 16, No. 9, 2006, pp. 910-918. doi:10.1111/j.1460-9592.2006.02018.x

[10] S. Suresh, et al., "Postoperative Pain Relief in Children Undergoing Tympanomastoid Surgery: Is a Regional Block Better than Opioids?” Anesthesia \& Analgesia, Vol. 94, No. 4, 2002, pp. 859-862. doi:10.1097/00000539-200204000-00015

[11] J. H. Shim, et al., "Ultrasound-Guided Greater Occipital Nerve Block for Patients with Occipital Headache and Short Term Follow up,” Korean Journal of Anesthesiology, Vol. 61, No. 1, 2011, pp. 50-54. doi:10.4097/kjae.2011.61.1.50

[12] K. J. Anand, "The Stress Response to Surgical Trauma: From Physiological Basis to Therapeutic Implications," Progress in Food \& Nutrition Science, Vol. 10, No. 1-2, 1986, pp. 67-132.

[13] K. J. Anand and P. R. Hickey, "Pain and Its Effects in the Human Neonate and Fetus," New England Journal of Medicine, Vol. 317, No. 21, 1987, pp. 1321-1329. doi:10.1056/NEJM198711193172105

[14] P. J. McGrath, et al., "American Academy of Pediatrics Report of the Subcommittee on Assessment and Methodologic Issues in the Management of Pain in Childhood Cancer,” Pediatrics, Vol. 86, No. 5, 1990, pp. 814-817.

[15] E. J. Hartley, et al., "Scalp Infiltration with Bupivacaine in Pediatric Brain Surgery,” Anesthesia \& Analgesia, Vol. 73, No. 1, 1991, pp. 29-32. doi:10.1213/00000539-199107000-00006

[16] S. Suresh, et al., "Does a Preemptive Block of the Great Auricular Nerve Improve Postoperative Analgesia in Children Undergoing Tympanomastoid Surgery?” Anesthesia \& Analgesia, Vol. 98, No. 2, 2004, pp. 330-333. 
doi:10.1213/01.ANE.0000097171.73374.AD

[17] B. Sung, et al., "Anesthetic Management with Scalp Nerve Block and Propofol/Remifentanil Infusion during Awake Craniotomy in an Adolescent Patient-A Case Report," Korean Journal of Anesthesiology, Vol. 59, pp. S179S182. doi:10.4097/kjae.2010.59.S.S179

[18] G. Pardey, et al., "Levobupivacaine Scalp Nerve Block in Children,” Pediatric Anesthesia, Vol. 18, No. 3, 2008, pp. 271-272. doi:10.1111/j.1460-9592.2008.02416.x

[19] H. J. Ahn, et al., "Effect of Preoperative Skull Block on Pediatric Moyamoya Disease,” Journal of Neurosurgery: Pediatrics, Vol. 2, No. 1, 2008, pp. 37-41.
doi:10.3171/PED/2008/2/7/037

[20] O. R. Adam and J. Jankovic, "Treatment of Dystonia,” Parkinsonism \& Related Disorders, Vol. 13, No. S3, 2007, pp. S362-S368. doi:10.1016/S1353-8020(08)70031-2

[21] J. Sebeo, et al., "Anesthesia for Pediatric Deep Brain Stimulation," Anesthesiology Research and Practice, Vol. 2010, 2010, Article ID: 401419. doi:10.1155/2010/401419

[22] B. Golianu, et al., "Pediatric Acute Pain Management," Pediatric Clinics of North America, Vol. 47, No. 3, 2000, pp. 559-587. doi:10.1016/S0031-3955(05)70226-1 